\title{
Tical: Chatbot sobre o Atlas Linguístico do Brasil no WhatsApp
}

\author{
Fábio Carlos Moreno ${ }^{1,3}$, Edio Roberto Manfio ${ }^{1,2}$, Cinthyan Renata Sachs \\ Camerlengo de Barbosa ${ }^{1}$, Jacques Duílio Brancher ${ }^{1}$ \\ ${ }^{1}$ Universidade Estadual de Londrina (UEL) \\ Caixa Postal 86057-970 - Londrina - PR - Brazil \\ ${ }^{2}$ Faculdade de Tecnologia Dep. Júlio Julinho Marcondes de Moura (Fatec) \\ Garça - SP - Brazil \\ ${ }^{3}$ Serviço Nacional de Aprendizagem Industrial (SENAI) \\ Santo Antônio da Platina - PR - Brazil \\ fbio_moreno@yahoo.com.br, prof.ediorobertomanfio@gmail.com, \\ \{cinthyan, jacques\} @uel.br
}

\begin{abstract}
Talking through mobile apps has become common nowadays and something essential and the WhatsApp is one of this App that stands out with thousands of users. It has such a great popularity that even some teachers use it as an extracurricular teaching methodology. Therefore, considering this accessibility advantage, a chatbot was developed to disseminate the Brazilian Linguistic Atlas, adding to the project, interdisciplinary features in Linguistics and Computer Science area, with its main focus on Natural Language Processing. The idea with this chatbot, in addition to show the Brazilian Atlas, is to stimulate other researchers' desire to develop other subjects that are related to education using the WhatsApp, which is a common tool among young people.
\end{abstract}

Resumo. Conversar através de aplicativos de celular se tornou comum e algo indispensável atualmente, e uns dos que se destacam com milhares de usuários para tal ação é conhecido por WhatsApp. Sua popularidade é tanta que alguns professores o utilizam como metodologia de ensino extraclasse. Sendo assim, aproveitando desta acessibilidade, foi desenvolvido um chatbot para a divulgação do Atlas Linguístico do Brasil, dando assim ao projeto, características de interdisciplinaridade na área de Linguística e Ciências da Computação, com o foco principal em Processamento de Linguagem Natural. A ideia com este chatbot, além de divulgar o Atlas do Brasil, é despertar em outros pesquisadores o desejo de desenvolver outros assuntos que sejam peculiar ao ensino, utilizando uma ferramenta comum entre os jovens que é o WhatsApp.

\section{Introdução}

O uso das TICs (Tecnologias da Informação e Comunicação) em salas de aula aumentam o desafio na aprendizagem e na metodologia de ensino [Bento e Cavalcante, 2013] e a utilização destes equipamentos motivam o surgimento de leis estaduais e 
municipais que proíbem seu uso e isto acontece em todo território nacional. Isso pode ser encontrado no sítio Jusbrasil [Jusbrasil, 2015].

Estados como Rio de Janeiro, Paraná, Minas Gerais, Ceará, Amazonas e Santa Cantarina são alguns exemplos que sancionaram leis que proíbem esta prática, com ressalvas para utilizações pedagógicas e com consentimentos do professor [Jusbrasil, 2015] [Celularcomcamera, 2014]. Porém, são muito poucos docentes que se utilizam deste recurso, como pode se notar na pesquisa realizada com professores do ensino médio de uma escola pública, em que apenas $5 \%$ utilizam ou permitem o uso do celular como parte da aula [Bento e Cavalcante, 2013].

A Organização das Nações Unidas para a Educação, Ciência e Cultura (Unesco) não só incentiva o uso em sala de aula como também afirma que o acesso a esses tipos de aparelhos facilitam na aprendizagem e auxilia o professor em escolas regulares, de ensino técnico e até na pré-escola [Barros, 2015]. Esta modalidade de ensino, a Mobile Learning, possibilita a aprendizagem em qualquer hora ou qualquer lugar, pois conectada entre alunos e professores pode-se recorrer a conteúdos dentro e fora da sala de aula [Barros, 2015] [Mühlbeier et. al., 2012].

Um exemplo desta ação já vem ocorrendo pelo intermédio dos próprios alunos que utilizam o aplicativo WhatsAppMesseger. Os estudantes se cadastraram em grupos com a finalidade de estudar e tirar dúvidas, como por exemplo, para provas de vestibulares. Alguns alunos com um conhecimento mais aguçado acabam dando aulas aos colegas neste ambiente [Moreno, 2014]. Além disso, estes tipos de prática atendem às expectativas dos professores e proporcionam oportunidades pedagógicas, contribuindo e motivando para a aprendizagem do aluno em diferentes áreas [Ferreira et. al., 2012] [Abech et. al., 2012] [Mühlbeier et. al., 2012].

Neste sentido, este artigo tem como objetivo apresentar o funcionamento do chatbot Tical - Tecnologia Interativa Conversacional sobre Assuntos Linguísticos - que funciona também por meio do WhatsApp e que responde questões da área de Linguística. Este sistema é um exemplo de uma boa prática de ensino dentro e fora de sala de aula, em que os alunos podem buscar e interagir para obter as respostas desejadas. É também de grande relevância por serem poucos os chatbots que respondem perguntas através deste aplicativo e por apresentar o diferencial de possibilitar consultas em âmbito global e sem custo a alunos, professores e pesquisadores.

\section{O WhatsApp}

O WhatsApp, criado por Brian Action e Jam Koum em 2009, é um software disponível para várias plataformas de celulares que permite trocar mensagens de texto, compartilhar vídeos, fotos e áudios e também fazer chamadas de voz a qualquer pessoa que possua uma conta no aplicativo [WhatsApp, 2015a].

Este app multiplataforma que funciona por meio da internet, já conta com um acesso de mais de meio milhão de pessoas ao redor do mundo. Estes índices têm crescido rapidamente nos países como o Brasil, México, Índia e Rússia. São mais de 700 milhões de fotos e 10 milhões de vídeos compartilhados e enviados por dia [WhatsApp, 2015b].

São vários os exemplos de sua utilização, o que torna deste app uma ferramenta realmente necessária e de resultados significativos. No blog dos criadores do WhatsApp 
[WhatsApp, 2015b], tem vários depoimentos que corroboram com isso, como é o caso de médicos da Índia que conseguem enviar fotos instantaneamente de pacientes que sofreram ataques cardíacos ou como nas montanhas de Madrid, em que socorristas o utilizam para encontrar e salvar caminhantes perdidos.

Com todos estes benefícios deste app, a proposta deste projeto é justamente apresentar a utilização de alguns destes recursos por meio de um robô de conversação que torna-se operacional usando uma linha comum de telefonia celular e um acesso simples à internet. A partir de então, vários conceitos vinculados ao ALiB - Atlas Linguístico do Brasil [Projeto ALiB, 2014] desde a sua gênese até perguntas específicas sobre a existência de uma carta lexical com as variantes para mexerica podem ser consultados.

\section{TICAL}

O chatbot Tical - Tecnologia Interativa Conversacional sobre Assuntos Linguísticos incluiu em seu desenvolvimento conceitos de Processamento de Linguagem Natural e tem como objetivo de difundir o ALiB (Atlas Linguístico do Brasil).

Este trabalho interdisciplinar conta com conceitos de Ciência da Computação, um banco de dados da área de Linguística e promove a difusão cultural do ALiB, que é o maior trabalho de pesquisa realizado na área de Geossociolinguística do Brasil até a atualidade [Cardoso, 2014a; 2014b].

Sua primeira versão foi desenvolvida em C++, porém algumas implementações e compatibilidades com outros componentes eram mais difíceis de serem feitas nesta plataforma. Por esse motivo, Tical (figura 1) foi reescrito em C\# para viabilizar inúmeras possibilidades, entre elas, sua implementação em WhatsApp.

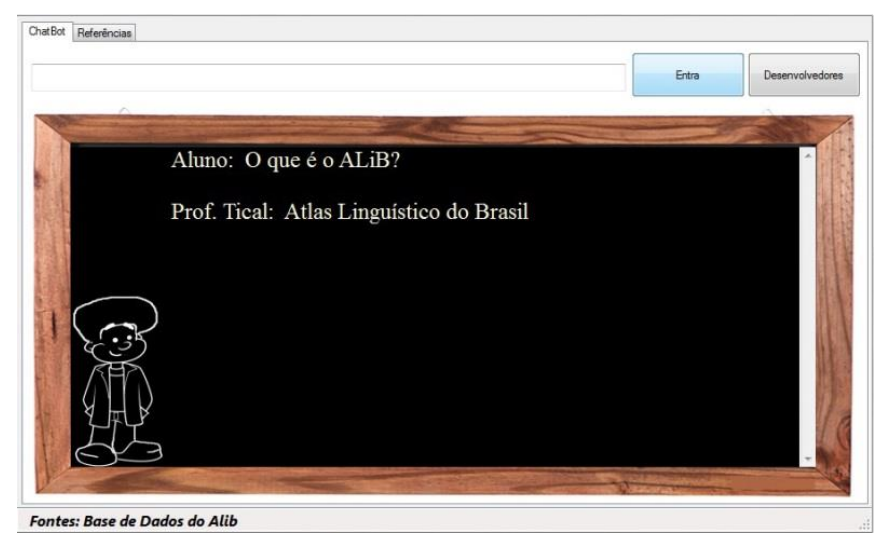

Figura 1. Interface de Tical em Desktop

Uma versão web também foi disponibilizada para testes no III CIDS - Congresso Internacional sobre Dialetologia e Sociolinguística [Manfio, Moreno e Barbosa, 2014b] para estudantes, professores e pesquisadores. Esta versão contou com a mesma estrutura do desktop, ou seja, apenas com uma interface para este ambiente (figura 2). 
CBIE-LACLO 2015

Anais do XXVI Simpósio Brasileiro de Informática na Educação (SBIE 2015)

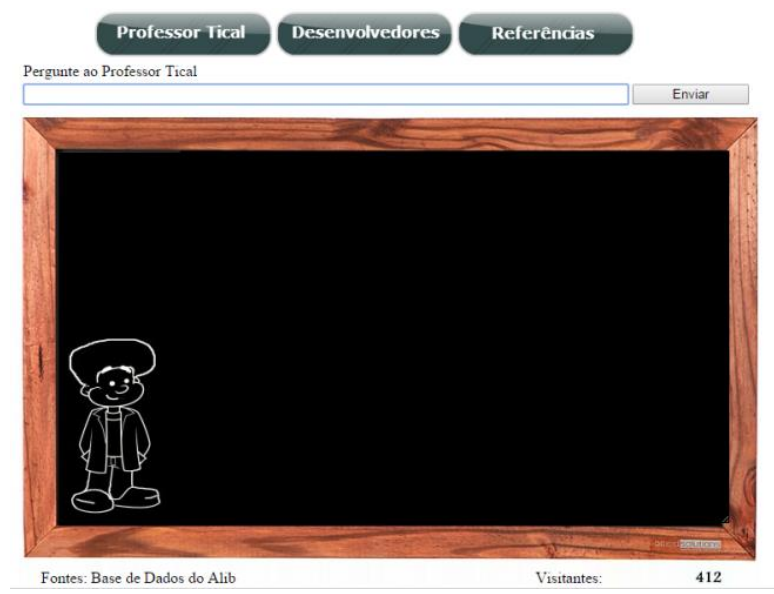

Figura 2. Interface de Tical na Web

Essa constante adequação das linguagens de programação e ferramentas quanto ao seu desenvolvimento/aprimoramento tem um papel fundamental na continuidade do projeto, uma vez que impacta diretamente na possibilidade de adequá-lo a novas plataformas de sistemas operacionais. Em outras palavras, a opção por reprogramá-lo ou reimplementá-lo utilizando a linguagem de programação C\# proporciona certa garantia de que estará sempre significativamente operacional, inclusive com as modificações que ainda serão lançadas.

Outro dado relevante vinculado a essa evolução para operacionalização em diferentes plataformas é que tanto os usuários quanto os desenvolvedores ganham em conhecimento: para aqueles, o conteúdo linguístico é mais acessível pois está disponível para diferentes dispositivos - móveis ou não - e em ambiência atualizadamente amigável; para estes, a busca pela adequação do aplicativo faz com que as soluções, sempre antecedidas por um desafio, sejam alcançadas mediante estudos teóricos e metodológicos cada vez mais aprimorados.

A base de dados do Tical, no caso perguntas/respostas e sinônimos/expressões equivalentes são cadastradas manualmente e esse procedimento tem sido feito por pessoas pertencentes à área envolvida do tema - $\mathrm{ALiB}$ - tendo em vista que, muitas das vezes, é o contexto que determina as relações sinonímicas ou de equivalência de significado [Manfio, Moreno e Barbosa, 2014a].

Para perguntas que não têm uma resposta cadastrada nesta base, Tical conta com um banco de dados para registrar estas questões que serão incrementadas posteriormente pelos desenvolvedores, além, é claro, daquelas perguntas existentes nos questionários [Comitê ALiB, 2001] elaborados e utilizados como guia pelo Comitê Nacional do Projeto ALiB durante as entrevistas com centenas de informantes de todo o Brasil.

Professor Tical apresenta em sua interface elementos relacionados com uma sala de aula, com o professor usando jaleco posicionado ao lado de um quadro negro que usa para tirar dúvidas em relação ao tema proposto.

Já para a versão no WhatsApp essa interface não é possível de ser realizada, porém todas as suas características de funcionamento foram implementadas. Na figura 3 pode-se verificar a interação com o Professor Tical no WhatsApp. 


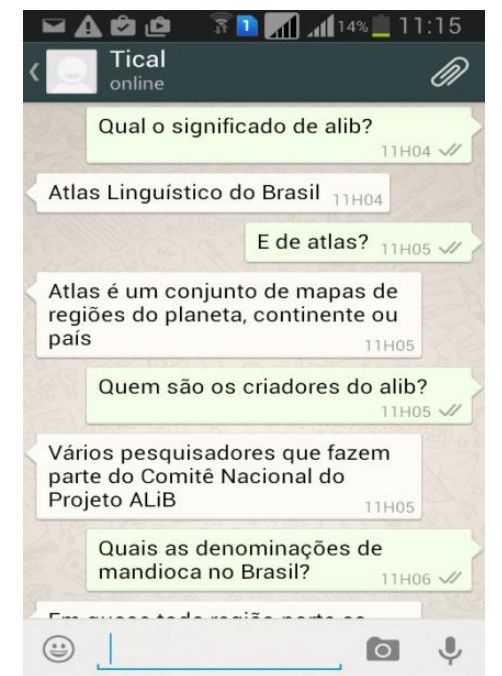

Figura 3. Interface com consultas feitas a Tical pelo WhatsApp

Em todas as interfaces de Tical vê-se o uso da linguagem natural, que é uma dos quatro estilos de interação juntamente com os menus, formulário e linguagem de comandos. Em outras palavras, Linguagem Natural é a utilização de texto escrito em idioma preestabelecido - no nosso caso, o português do Brasil - a partir de um prompt [Barbosa, 2004].

Válido salientar que chatbots voltados à educação, aprendizado e difusão cultural, de um modo geral com interfaces e funções similares a Tical, não são novidades. Devido à popularidade dos smartphones e também de sua capacidade de processamento, há uma tendência para o surgimento de robôs similares. O Mobile Bot [Andrade, 2012], por exemplo, feito em linguagem Java em um banco AIML, auxilia no compartilhamento de conhecimento, no esclarecimento de dúvidas e na aprendizagem à distância. Outro exemplo é o Geranium [Griol e Callejas, 2013], cuja proposta prevê uma estrutura modular para desenvolver um sistema amigável, fácil de usar e eficiente, com o qual os alunos podem interagir utilizando, como sempre, a Linguagem Natural.

O Robô ED, por sua vez, pertencente ao Conpet [Conpet, 2015] e ligado à Petrobras e ao Ministério de Minas e Energia, 'conversa' sobre assuntos ligados ao meio ambiente e opera totalmente em português do Brasil. Há também o Instant Message us [Allison, 2012], semelhante ao Robo Ed, porém com mais recursos, o EFL Classroom com conceitos de Interação de Linguagem Natural (NLI) projetados para simular uma conversa [Allison, 2012].

Tical, entretanto, tem suas particularidades e vantagens: é um dos únicos que versam sobre Linguística; está todo em Português do Brasil; terá interação por voz com estudantes que poderão enviar suas perguntas sem a necessidade da digitação de texto; operará com retorno das respostas em formato de áudio com voz sintetizada; efetuará gravação do conteúdo do chat para uma melhor interação com usuário.

\section{Implementação e Estrutura do Tical}

O armazenamento das informações é feito através de dois arquivos XML, sendo um para as perguntas e respostas e outro para sinônimos. Tical tem em sua estrutura conceitos de tabelas hash encadeadas, utilizando-se da função de Jenkins [Jenkins, 1997] para fazer os cálculos necessários para o procedimento. 
Tical utiliza-se da linguagem natural e para isso temos um arquivo de perguntas e repostas, no qual as chaves são as palavras em evidências para um cálculo hash que identificará a posição na tabela e consequentemente o retorno da consulta. A pergunta "Como se chama macaxeira no Brasil?", por exemplo, é colocada em ordem e mantida somente as palavras com maior relevância, que para Amorim, Cury e Menezes (2012) são stopwords, ou seja, irrelevantes para o conjunto a ser aplicado em uma consulta. $\mathrm{O}$ Quadro 1 exibe as palavras-chave da frase "Como se chama macaxeira no Brasil?" em ordem e sem a substituição dos sinônimos.

Quadro 1 - Chave Pergunta e Resposta - sem atribuir os sinônimos

? BRASIL CHAMA MACAXEIRA

Note que no exemplo da Tabela 1, o texto está em ordem alfabética para não afetar no cálculo do hash e o sinal de interrogação é importante para definir se é um questionamento ou uma afirmação.

Já o arquivo XML de sinônimos permite que questionamentos possam ser construídos de diferentes maneiras de forma a expandir a consulta e melhorar a recuperação da informação [Amorim et. al., 2012]. A tabela 1 mostra algumas variantes para a pergunta "Como se chamam macaxeira no Brasil".

Tabela 1 - Variantes para a pergunta "Como se chama macaxeira no Brasil?"

\begin{tabular}{|l|l|}
\hline Como denomina mandioca no Brasil? & Quais as denominações para aipim no Brasil? \\
\hline Quais os nomes de mandioca no Brasil? & Quais outros nomes para aipim no Brasil? \\
\hline Como denomina mandioca no Brasil? & Quais os nomes de macaxeira no Brasil? \\
\hline Quais as denominações para mandioca no Brasil? & Como denomina macaxeira no Brasil? \\
\hline Quais outros nomes para mandioca no Brasil? & Quais as denominações para macaxeira no Brasil? \\
\hline Quais os nomes de aipim no Brasil? & Quais outros nomes para macaxeira no Brasil? \\
\hline Como denomina aipim no Brasil? & Nomes para a macaxeira no Brasil? \\
\hline
\end{tabular}

São várias combinações possíveis de serem construídas e que dificultaria o trabalho de cadastro e manutenção do chatbot. No entanto, a tabela de sinônimos reduz e facilita este processo, quando alguma pergunta é feito a Tical. $\mathrm{O}$ primeiro processo a ser realizado é a busca por sinônimos, ilustrado na Tabela 2, e quando o equivalente é encontrado, é substituído na frase principal.

Tabela 2 - Tabela de sinônimos para pergunta "Como se chama macaxeira no Brasil?"

\begin{tabular}{|l|l|}
\hline \multicolumn{1}{|c|}{ Palavra Chave } & \multicolumn{1}{c|}{ Sinônimos } \\
\hline \multirow{2}{*}{ MANDIOCA } & MACAXEIRA \\
\cline { 2 - 2 } & AIPIM \\
\hline \multirow{4}{*}{ NOMES } & DENOMINA \\
\cline { 2 - 2 } & CHAMAM \\
\cline { 2 - 2 } & DENOMINAÇÃO \\
\cline { 2 - 2 } & NOME \\
\cline { 2 - 2 } & DENOMINA \\
\hline
\end{tabular}

Quando todas as substituições forem realizadas, a frase fica com o seguinte formato: "Como se nomes mandioca no Brasil?". Esta é colocada em uma lista ordenada e feita todas as combinações possíveis. Ao final, a combinação com maior quantidade de chaves encontrada retorna a resposta equivalente (Tabela 3 ). 
CBIE-LACLO 2015

Anais do XXVI Simpósio Brasileiro de Informática na Educação (SBIE 2015)

Tabela 3 - Chave e Retorno

\begin{tabular}{|c|c|l|}
\hline $\begin{array}{c}\text { Maior Quantidades } \\
\text { de Chaves } \\
\text { encontrada }\end{array}$ & $\begin{array}{c}\text { Combinação com maior quantidades } \\
\text { de Chaves }\end{array}$ & \multicolumn{1}{|c|}{ Retorno do Tical } \\
\hline 4 & ? BRASIL NOMES MANDIOCA & $\begin{array}{l}\text { Em quase toda região norte as pessoas } \\
\text { chamam-na de 'macaxeira'. No centro- } \\
\text { oeste e cidade de São Paulo predomina } \\
\text { o termo 'mandioca'. Na Bahia e nas } \\
\text { capitais Vitória, Rio de Janeiro, } \\
\text { Florianópolis e Porto Alegre é } \\
\text { conhecida como 'aipim' }\end{array}$ \\
\hline
\end{tabular}

Como dito anteriormente, esta versão de Tical foi desenvolvida em C\# e para que operasse no WhatsApp, foi necessário o uso da ferramenta WhatsAPInet, que é desenvolvida em mesma linguagem e tem seu código aberto. Tical está em funcionamento 24 horas por dia em um servidor configurado na Universidade Estadual de Londrina (UEL). A figura 4 é ilustra o funcionamento.
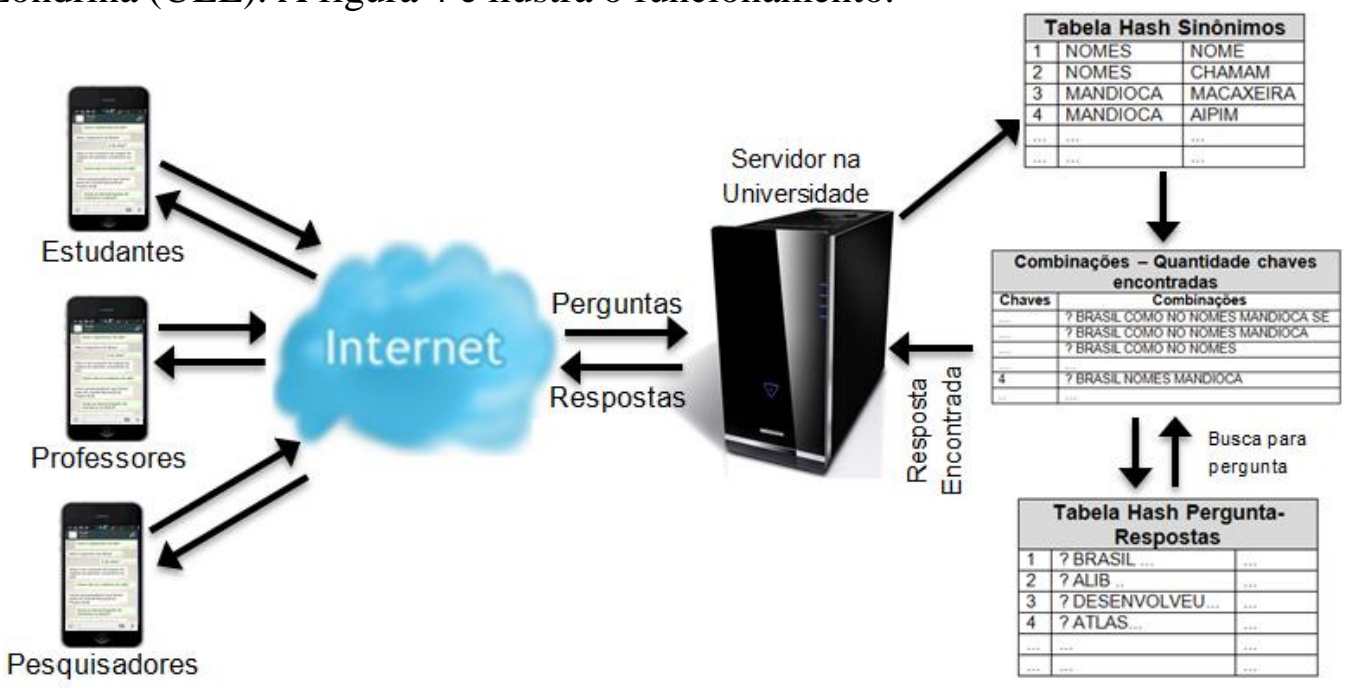

Figura 4 - Estrutura Tical no WhatsApp

Para esta versão, foram limitadas as quantidades de palavras para questionamentos, pela razão que textos escritos de maior extensão geraram problema em função do modo como o hash ordena suas listas, ocasionando lentidão. Então, decidiu-se limitar a quantidade de palavras a ser consultada por Tical para quinze, considerando que a função dos chatbots, de um modo geral, é dar conta de perguntas mais sucintas e objetivas. Em caso de perguntas contendo um número de palavras maior que o padrão definido, a resposta será "Refaça a pergunta de forma mais simplificada".

\section{Resultados}

Tal como colocado há pouco, Tical em formato web manteve a mesma estrutura de funcionamento e foi apresentado e disponibilizado para consulta no III CIDS [Manfio; Moreno; Barbosa, 2014b] e todos os erros e respostas não respondidas foram catalogadas para análises.

Desde o início do III CIDS até o momento que este artigo estava sendo escrito, foram 408 acessos, somando um total de 734 perguntas, dos quais 107 foram catalogadas com problemas como mostra a tabela 4. 
Tabela 4 - Erros em Tical

\begin{tabular}{|c|c|c|c|}
\hline Erros de português & Fora do contexto & $\begin{array}{c}\text { Perguntas sem respostas } \\
\text { cadastradas }\end{array}$ & $\begin{array}{c}\text { Erro na elaboração da } \\
\text { pergunta }\end{array}$ \\
\hline 08 & 28 & 43 & 28 \\
\hline
\end{tabular}

Para esta versão em WhatsApp, as 43 perguntas não respondidas foram implementadas para que o chatbot conseguisse dar estas respostas corretamente. Perguntas fora do contexto demonstradas na tabela 5 não foram trabalhadas neste momento, porém serão tratadas em projetos futuros.

Tabela 5 - Frases fora do contexto

\begin{tabular}{|l|l|}
\hline Olá? & Quem é você? \\
\hline Como funciona? & O que é chuva? \\
\hline Professor, o senhor é burro? & Onde fica o Brasil? \\
\hline
\end{tabular}

Em seu formato web, o Tical conseguiu retornar 627 respostas dos 734 questionamentos o que significa um aproveitamento de $85 \%$. Considerando que este trabalho ainda é um protótipo isto representa bons resultados em questões de interação. As versões Web e WhatsApp se mantém funcionando separadamente, porém com a mesma estrutura backend já com todas as correções citadas.

Migrando de um ambiente essencialmente acadêmico de ensino superior para um mais técnico educacional, Tical foi também testado por 19 alunos do Ensino MédioProfissional da faixa etária média de 16,8 anos do Centro Integrado SESI/SENAI/IEL de Santo Antônio da Platina, na cidade do Paraná, onde um dos autores leciona. Após utilizar o celular para trocar mensagens com o Tical, os alunos responderam a um questionário, o qual deram notas de 0 a 5 para cada pergunta, onde zero representa menor incidência e cinco uma maior incidência, gerando os seguintes resultados:

Tabela 6 - Pesquisa e Aplicabilidade

\begin{tabular}{|l|l|}
\hline frequência da utilização de celular/tablet para fins didáticos dos alunos em sala de aula & 2,0 pontos \\
\hline utilização destes equipamentos para estudos em casa & 4,42 pontos \\
\hline aprovação por parte dos que utilizariam a ferramenta para estudos em sala e extraclasse. & 4,8 pontos \\
\hline
\end{tabular}

Através dos dados apresentados na tabela 6, observa-se uma margem de 2 pontos para a utilização de celulares/tablet pelos alunos em salas de aulas para fins didáticos, e uma pontuação que foi o dobro $(4,42)$ demostrando que eles não usam só este equipamentos para entretenimento, mas também para estudos extraclasses. O chatbot Tical foi aprovado pelos alunos com uma pontuação média de 4,8, e também responderam que gostariam de utilizar chats como este para estudos em sala e extraclasse.

E relevante observar que, paralelamente à construção deste texto, outra pesquisa estava sendo conduzida, a qual se trata da inclusão de todas as perguntas utilizadas nos Questionários do ALiB [Comitê, 2001] no banco de dados de Tical que são mais de 300 perguntas. A tarefa consiste em fazer com que Tical se torne cada vez mais apto a responder sobre variantes fonéticas e lexicais de todo o país.

A palavra pipa, por exemplo, da pergunta de número 158 do Questionário [Comitê, 2001], apresenta mais de 10 variantes em território nacional: papagaio, curica, raia, periquito, ratinho, avião, bolachinha, caixotinho, capocheta, jereco, pandorga [Cardoso, 2014b]. Isso significa, no mínimo, mais de 10 entradas no aplicativo para a mesma explicação/resposta. A dimensão de nuances para essa mesma reposta, no 
entanto, pode aumentar bastante em função da acurácia que se deseja. Portanto, note-se que o estudo aqui realizado é apenas o primeiro passo de uma grande caminhada a ser feita em termos de implementação.

\section{Conclusões}

Neste artigo apresentamos o chatbot Tical que responde a perguntas sobre o Atlas Linguístico do Brasil e disponibiliza aos alunos outras maneiras de ampliar seu aprendizado como defende Abech (2012). Através desta estrutura, outros temas podem ser desenvolvidos, como é o caso do Carolina - Conversação Automática por Robô sobre Literatura Nacional e Afins - em desenvolvimento pelos autores deste artigo, que é por enquanto um protótipo que responde a perguntas sobre Literatura Brasileira e que pode ser utilizado por docentes em sua metodologia de ensino.

Umas das grandes vantagens de Tical, em relação aos outros chatbots deste tipo, como ressaltado ao longo desse trabalho, é que ele funciona através de um messenger conhecido mundialmente [WhatsApp, 2015b] e, por esta razão, não tem necessidade de realizar qualquer instalação ou configuração adicional, sendo apenas indispensável adicioná-lo aos contatos de seu aparelho celular.

Algumas aplicabilidades ainda em fase de testes são as seguintes: i) a interação por voz e vídeo a uma parcela dos estudantes que poderão enviar suas perguntas sem a necessidade da digitação de texto; ii) retorno das respostas em formato de áudio com voz sintetizada; iii) gravação do chat para uma melhor interação com usuário.

\section{Referências}

Abech, M. et al. (2012) "Um Modelo de Adaptação de Objetos de Aprendizagem com foco em Dispositivos Móveis", Anais do Simpósio Brasileiro de Informática na Educação, Vol. 23, No. 1.

Allison, D. (2012) “Chatbots in the library: is it time?", Library Hi Tech,Vol. 30(1), p. 95-107.

Amorim, M. T. C. F. de, Cury, D., Menezes, C. S. (2012) "Um helpdesk inteligente baseado em ontologias", Anais do Simpósio Brasileiro de Informática na Educação, Vol. 23, No. 1.

Andrade, R. M. (2012) "Mobile bot: um chatterbot educacional para dispositivos móveis", Revista Brasileira de Computação Aplicada, v. 4(2), p. 83-91.

Barros, M. L. (2015) "Unesco estimula o uso de celulares em salas de aula", Disponível em http://odia.ig.com.br/noticia/rio-de-janeiro/2015-02-21/unescoestimula-uso-de-celulares-em-salas-de-aula.html

Barbosa, C. R. S. C. de. (2004) "Técnicas de parsing para gramática livre de contexto lexicalizada da língua Portuguesa" (Tese de Doutorado), Curso de Engenharia Eletrônica e Computação, Instituto Tecnológico de Aeronáutica, São José dos Campos, $171 \mathrm{f}$.

Bento, M. C. M., Cavalcante, R. S. (2013) "Tecnologias Móveis em Educação: o uso do celular na sala de aula", Educação, Cultura e Comunicação, v. 4, n. 7.

Cardoso, S. A. M. S. et al. (2014a) "Atlas Linguístico do Brasil: Introdução”, Vol. 1. Londrina: Eduel. 
Cardoso, S. A. M. S. et al. (2014b) “Atlas Linguístico do Brasil: Cartas Linguísticas I", Vol. 2, Londrina: Eduel.

Celularcomcamera (2014) "Uso de Celulares em sala de aula proibido por lei", Disponível em http://www.celularcomcamera.com.br/uso-de-celulares-em-sala-deaula-proibido-por-lei/ .

Conpet (2015), Programa Nacional da Racionalização do uso dos derivados do petróleo e do gás natural - Robô Ed. Disponível em: http://www.ed.conpet.gov.br/br/converse.php.

Comitê Nacional do Projeto ALiB (2001) "Atlas Linguístico do Brasil: questionário 2001/Comitê Nacional do Projeto ALiB”, Londrina: Editora UEL.

Ferreira, L. G. A., Gluz, J. C., Barbosa, J. L. V. (2012) "Um Modelo Multiagente para Recomendação de Conteúdo Educacional em um Ambiente Ubíquo", Anais do Simpósio Brasileiro de Informática na Educação, Vol. 23, No. 1.

Griol, D., Callejas, Z. (2013) "An Architecture to Develop Multimodal Educative Applications with Chatbots", International Journal of Advanced Robotic Systems, Vol. 10.

Jenkins, B. (1997) “Algorithm alley: Hash functions”, Dr. Dobb's Journal of Software Tools.

Jusbrasil (2015) "Legislação para Uso de Telefone Celular em Sala de Aula", Disponível em http://www.jusbrasil.com.br/legislacao/busca?q=Uso+de+ Telefone+Celular.

Manfio, E. R., Moreno, F. C., Barbosa, C. R. S. C. de. (2014a) "Tecnologia Interativa Conversacional sobre Assuntos Linguísticos - Tical: Linguagem e Significação", In: IX Seminário de Estudos sobre Linguagem e Significação e X Simpósio de Leitura da UEL "Convenções e Ousadias da Linguagem", Caderno de Resumos, Londrina: UEL, p. 54-55.

Manfio, E. R., Moreno, F. C., Barbosa, C. R. S. C. de. (2014b). "Professor Tical: robô de conversação sobre dialetologia e geossociolinguística", III CIDS - Congresso Internacional de Dialetologia e Sociolinguística - Variação, Atitudes Linguísticas e Ensino, Londrina, UEL. Caderno de Resumos, ISBN: 978857846297 0, Londrina, p. 48.

Moreno, A. C. (2014) "Candidatos do Enem estudam para as provas em grupos no WhatsApp”, Disponível em http://g1.globo.com/educacao/enem/2014/noticia/2014/ 10/ candidatos-do-enem-estudam-para-provas-em-grupos-no-whatsapp.html.

Mühlbeier, A. R. K et al. (2012) "MOBILE HQ: O Uso de Softwares Educativos na Modalidade M-Learning", Anais do Simpósio Brasileiro de Informática na Educação, Vol. 23, No. 1.

Projeto ALiB. (2014) “Atlas Linguístico do Brasil”, Disponível em http://twiki.ufba.br/ twiki/bin/view/Alib/WebHome.

WhatsApp (2015a) "WhatsApp", Disponível em http://www.whatsapp.com.

WhatsApp (2015b) “Blog do WhatsApp”, Disponível em http://blog.whatsapp.com. 